\title{
Invasive Cervical Resorption: A Clinical Case Report demonstrating the Value of CBCT in Diagnosis and Treatment Planning
}

\author{
Venkat Canakapalli
}

\begin{abstract}
Invasive cervical resorption (ICR) is a common clinical entity. Invasive cervical resorption can be arrested using the "Heithersay approach" (i.e., mechanical debridement, treatment with TCA, and restoration). Prudent case selection and proper execution can lead to the successful treatment and long-term retention of the tooth. The key aspects are the location, size, and accessibility of the lesion and the structural integrity of the tooth. Cone beam computed tomography imaging is an invaluable tool to assess and treat ICR.
\end{abstract}

Keywords: Cervical resorption, Cone beam computed tomography, Heithersay Approach, Invasice cervial resorption.

How to cite this article: Canakapalli V. Invasive Cervical Resorption: A Clinical Case Report demonstrating the Value of CBCT in Diagnosis and Treatment Planning. J Oper Dent Endod 2016;1(2):87-89.

Source of support: Nil

Conflict of interest: None

\section{INTRODUCTION}

Invasive cervical resorption (ICR) is a common clinical entity. Although common, ICR is not well understood within the dental community and is often undiagnosed or misdiagnosed. Even when diagnosed correctly, there is often disagreement or confusion about the best course of treatment, even within the endodontic community. Invasive cervical resorption invades the tooth from the periodontal ligament, apical to the epithelial attachment and is not evident clinically in the early stages. In many cases, it is first detected radiographically or when the tooth takes on a pink appearance because of deep red granulation tissue showing through the tooth structure. ${ }^{1-3}$

On periapical conventional radiographs, ICR may be a barely discernible radiolucency or dramatically evident. The lesions vary from well-delineated radiolucencies that are quite obvious to poorly defined lesions with irregular

Private Practitioner

Private Practice Limited to Endodontics, Tauranga, New Zealand

Corresponding Author: Venkat Canakapalli, Private Practitioner, Private Practice Limited to Endodontics, Tauranga New Zealand, e-mail: endovc@gmail.com borders and sometimes resemble caries radiographically. When ICR is superimposed in the pulp space, pulp space anatomy is usually evident.

Invasive cervical resorption is often seen in the cervical area of the tooth, but because it is initiated apical to the epithelial attachment, it can present anywhere in the root. In the early stages, it may be somewhat symmetrical, but the larger lesions tend to be asymmetrical.

Heithersay wrote a classic series of articles in which he described the features, possible predisposing factors, and recommended treatment regimen for ICR. He described his treatment regime, which included mechanical and chemical debridement of the resorptive lesions, followed by restoration, and analyzed the treatment results. For the small, localized lesions (class 1 or 2), he reported that successful treatment was close to $100 \%$. For the moderatesize lesions (class 3 ), he reported a $77.8 \%$ success rate. For the extensive, class 4 lesions, his success rate was only $12.5 \%$ (Fig. 1).

Much of the literature pertaining to treatment of ICR is in the form of case reports and lacks long-term followup. In 2010, Schwartz et al published three case reports with recalls of 4,8 , and 9.5 years.

\section{TREATMENT PLANNING}

When ICR is diagnosed, there are generally three choices for treatment: (1) No treatment with eventual extraction when the tooth becomes symptomatic; (2) immediate extraction; or (3) access, debridement, and restoration of the resorptive lesion.

Computed tomography (CT) scanning can be very useful in treating ICR. Periapical radiographs tend to underestimate the size of the resorptive lesion. Computed tomography scanning gives a more accurate estimation of lesion size and, in some cases, may discourage treatment. Computed tomography images also provide the precise location of the lesion in three dimensions and the relationship to the surrounding bone, which can be very helpful in decision making.

\section{CASE REPORT}

A 43-year-old Caucasian woman was referred to the author's practice for opinion and treatment of tooth 


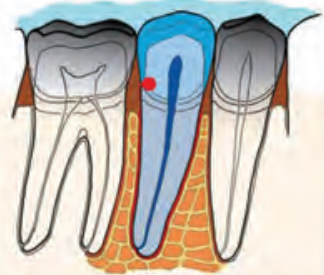

Class 1

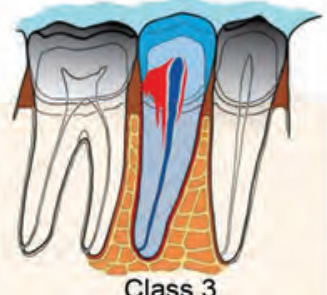

Class 3

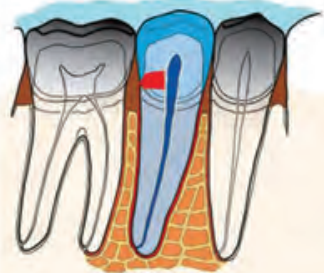

Class 2

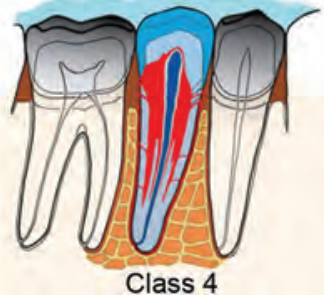

Class 4
Fig. 1: Classification of invasive cervical resorption

\#23. Her restorative dentist noted a moderate-size oval radiolucency in the cervical area with no symptoms and referred her for evaluation (Fig. 2). He noted a pink discoloration on the palatal surface of the tooth (Fig. 3). His tentative diagnosis was internal resorption. Patient had a history of orthodontic treatment as a teenager and could not recall a history of trauma or any of the other possible predisposing factors.

Endodontic testing found that the tooth was nontender to pressure and percussion and responded normally to cold compared with the adjacent teeth. There were no significant periodontal pockets.

Cone beam computed tomography (CBCT) scan by CS 9000 3D (Carestream Health) provided precise information about the size, location, and extension of the ICR lesion (Figs 4 and 5). The endodontic diagnosis was normal pulp and normal periapex. The radiolucent lesion was diagnosed as ICR. As the pulp was not involved and the lesion was supracrestal, the following treatment options were discussed with the patient. These included (1) no treatment with eventual extraction of the tooth when it becomes symptomatic; (2)

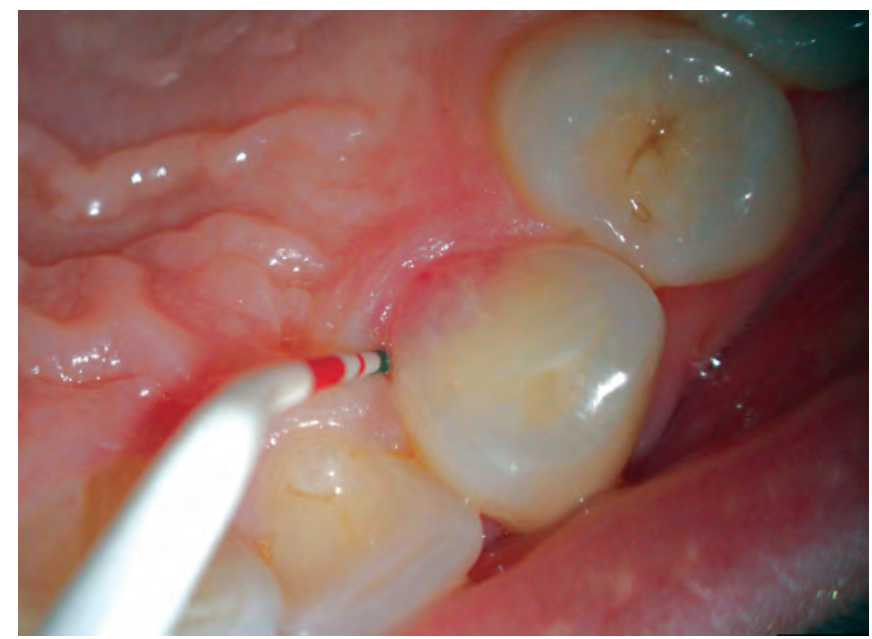

Fig. 3: Preoperative clinical evaluation

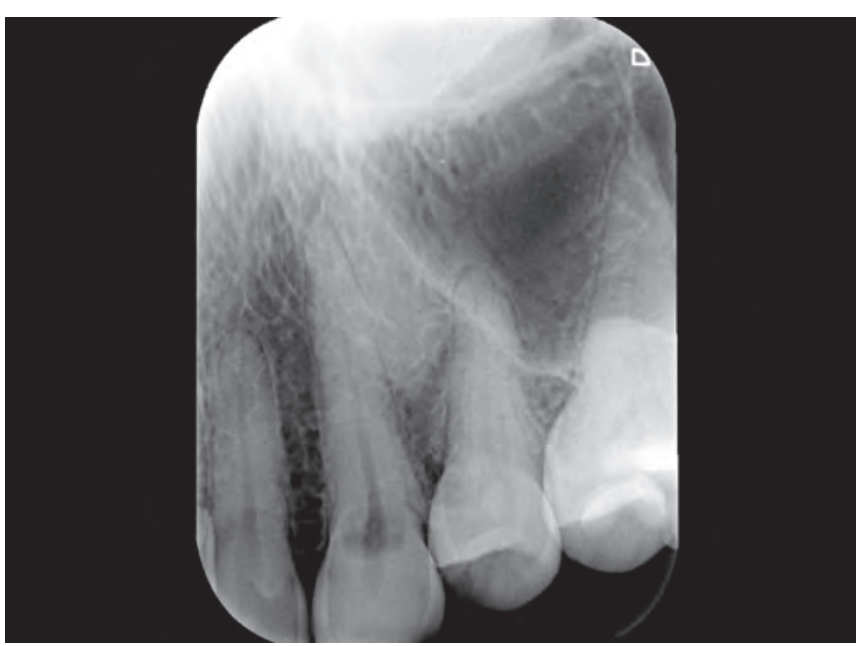

Fig. 2: Preoperative radiograph

surgical exposure of the lesion, debridement, and restoration with or without endodontic treatment. After some discussion, the patient decided on option \#2.

The lesion was accessed without elevating a flap and debrided with a carbide round bur in a slow-speed handpiece (Fig. 6). All the obvious resorptive tissue was removed until smooth, clean dentin was present. The dentin was then scrubbed for 1 minute with $90 \%$ aqueous trichloroacetic acid (TCA) and a cotton ball. Trichloroacetic acid is very caustic and cauterizes the residual resorptive tissue, which makes it more obvious under magnification (Fig. 7). The defect was very close but did not expose the pulp, and root canal treatment was not performed considering the tooth was asymptomatic. Dentin surface was "refreshed" with the round bur in preparation for restoration placement. GC Fuji IX GP FAST (GC America) was used to restore the defect (Fig. 8). Patient was informed that pulp could become symptomatic in the future and an endodontic procedure may become necessary at that stage. At 1-year review, the pulp responded normally with good soft tissue healing (Figs 9 to 11). ${ }^{4-6}$

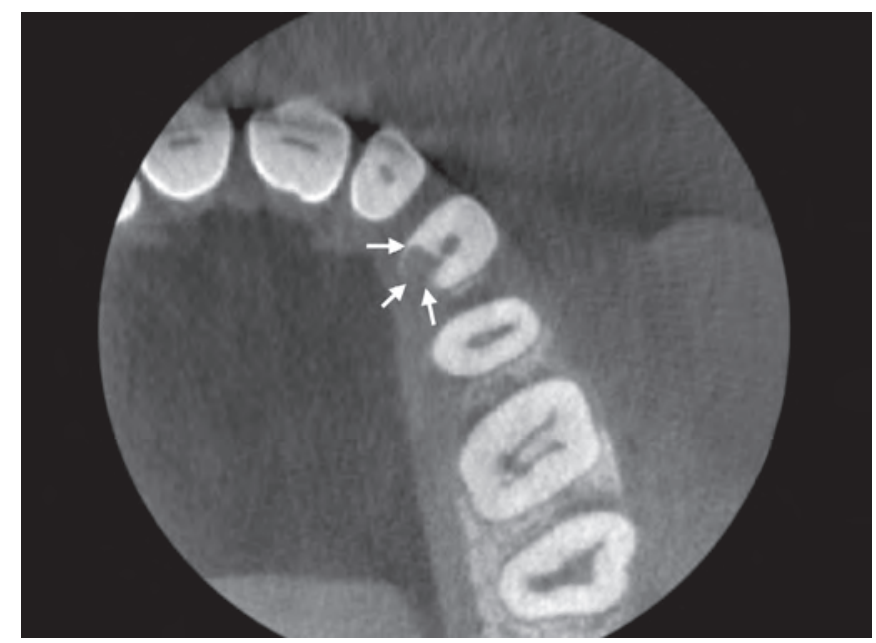

Fig. 4: Invasive cervical resorption - СВСТ cross-sectional image 


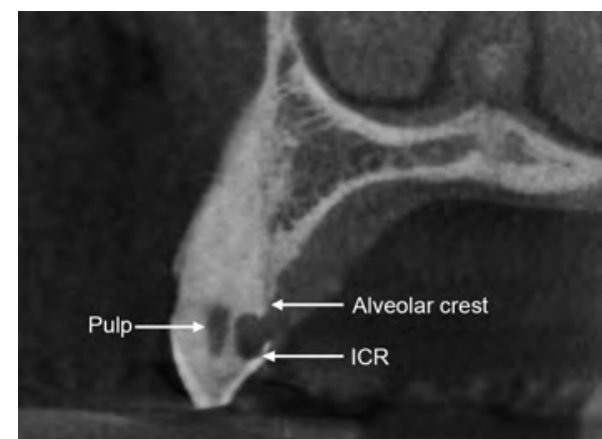

Fig. 5: Appearance of ICR in longitudinal section of CBCT

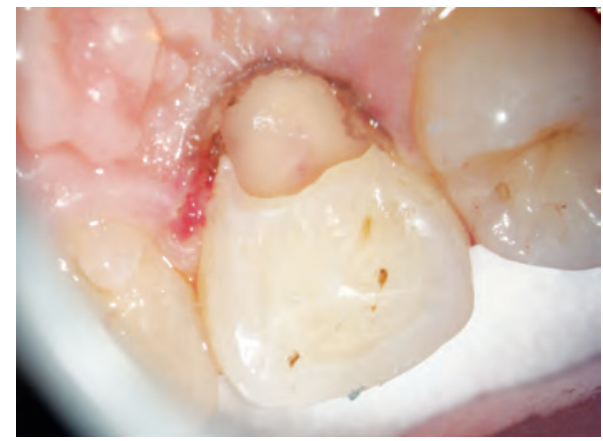

Fig. 7: Lesion debridement

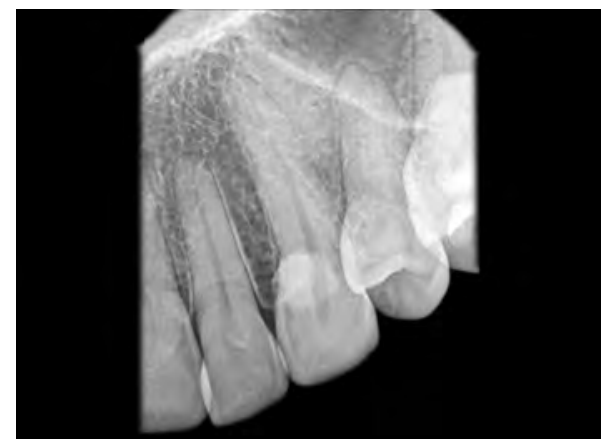

Fig. 9: Postoperative radiograph

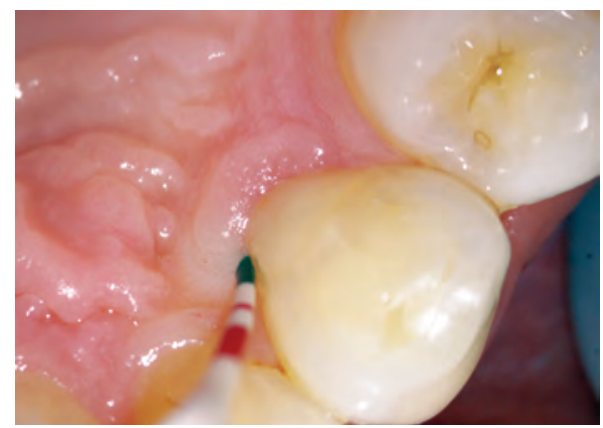

Fig. 11: Postoperative clinical evaluation - 1 year follow-up

\section{CONCLUSION}

Invasive cervical resorption can be arrested using the "Heithersay approach" (i.e., mechanical debridement, treatment with TCA, and restoration). Prudent case selection and proper execution can lead to the successful treatment and long-term retention of the tooth. The key aspects are the location, size, and accessibility of the lesion and the structural integrity of the tooth. Cone beam

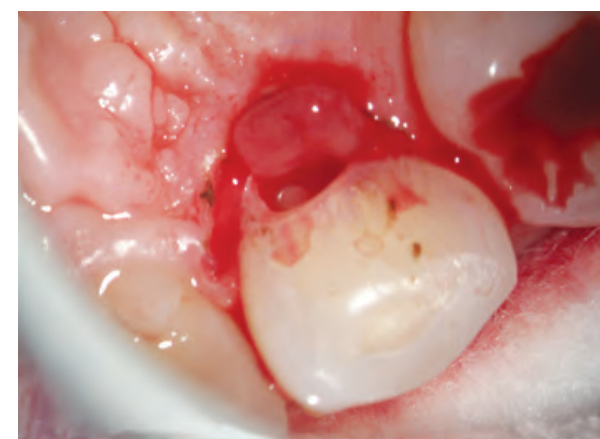

Fig. 6: Soft tissue defect in ICR

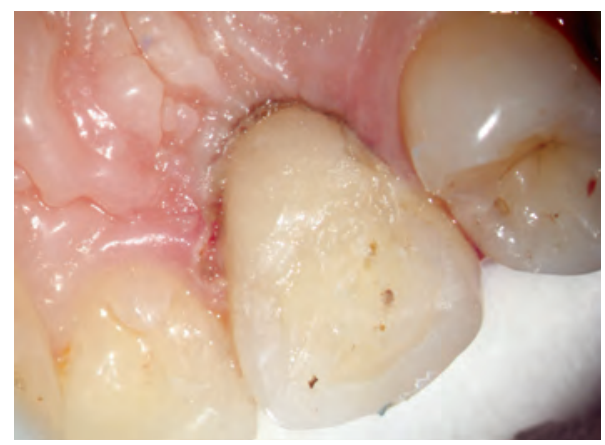

Fig. 8: GIC restoration

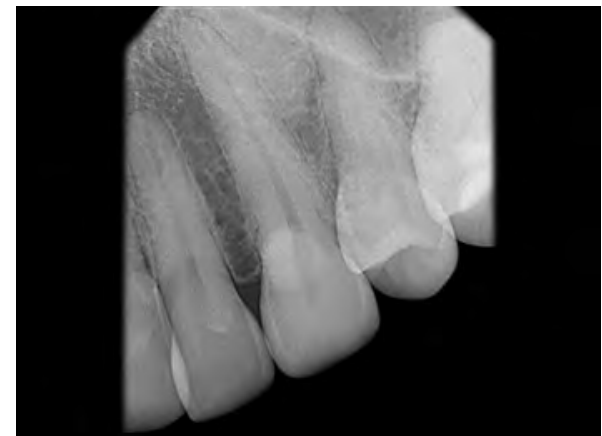

Fig. 10: One year review radiograph

computed tomography imaging is an invaluable tool to assess and treat ICR.

\section{REFERENCES}

1. Heithersay GS. Clinical, radiologic and histopathologic features of invasive cervical resorption. Quintessence Int 1999 Jan;30(1):27-37.

2. Heithersay GS. Invasive cervical resorption. Endod Topics 2004 Mar;7(1):73-92.

3. Patel S, Kanagasingam S, Pitt Ford T. External cervical resorption: A review. J Endod 2009 May;35(5):616-625.

4. Bergmans L, Van Cleynenbreugel J, Verbeken E, Wevers M, Van Meerbeek B, Lambrechts P. Cervical external root resorption in vital teeth. X-ray micro focus tomographical and histopathological case study. J Clin Periodontol 2002 Jun;29(6):580-585.

5. Heithersay GS. Invasive cervical resorption: an analysis of potential predisposing factors. Quintessence Int 1999 Feb;30(2):83-95.

6. Heithersay GS. Treatment of invasive cervical resorption: An analysis of results using topical application of trichloracetic acid, curettage, and restoration. Quintessence Int 1999 Feb;30(2):96-110. 